\title{
Infezione da HCV nel paziente in lista d'attesa e nel trapianto di rene: la nostra esperienza
}

\author{
A. Schena \\ D.E.T.O., Sezione di Nefrologia, A.O. Policlinico Consorziale di Bari, Bari
}

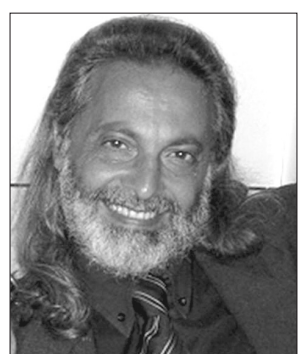

Secondo i dati riportati in letteratura e ormai universalmente dimostrati, l'infezione da $\mathrm{HCV}$ non rappresenta una controindicazione al trapianto di rene. Nella nostra esperienza, non risulta significativa differenza, in termini sia di patient sia di graft survival tra riceventi $\mathrm{HCV}+\mathrm{e}$ riceventi HCV- (Figg. 1 e 2). Tuttavia, è auspicabile, nei pazienti in lista d'attesa per trapianto renale (LAT) un'attenta valutazione clinicoistologica dell'epatopatia e, laddove indicato, l'attuazione di un programma terapeutico antivirale.

In quest'ottica, nel nostro Centro, è attivo un programma specifico, condotto in collaborazione con i colleghi della U.O. di Gastroenterologia Universitaria. Sui nostri pazienti $\mathrm{HCV}+$ in $\mathrm{LAT}$ sono state effettuate 46 biopsie epatiche per la valutazione del grading (percentuale di necrosi ed infiammazione) e dello staging (percentuale di fibrosi) dell'epatopatia, in associazione alla determinazione dell' $\mathrm{HCV}$ RNA (qualitativa e quantitativa), del

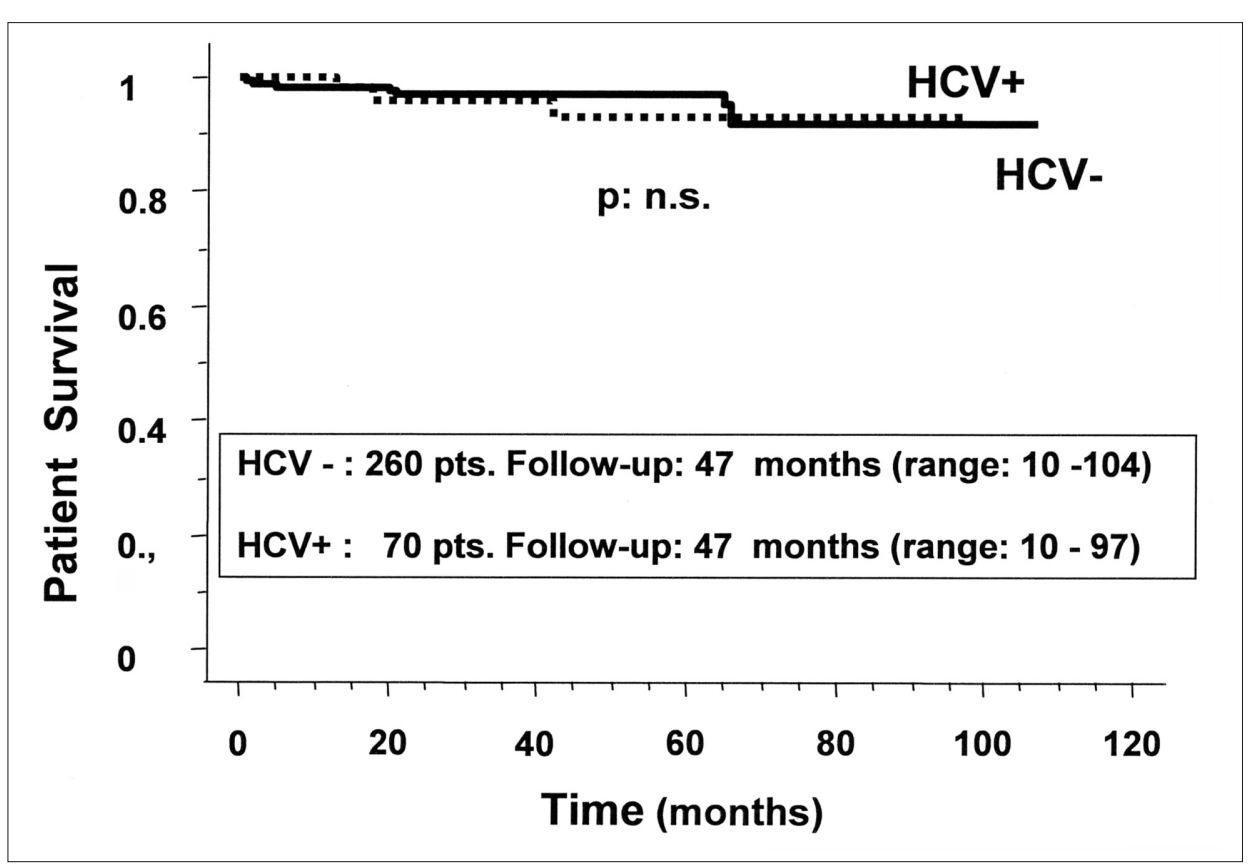

Fig. 1

genotipo e degli indici di funzione epatica. Sulla base dei quadri istologici risulta, in questi pazienti, una netta prevalenza dei gradi 1 e $2(43 \%$ e $52 \%$, rispettivamente) e degli stadi 1 e $2(57.2 \%$ e $33.3 \%$, rispettivamente). In altre parole, si evidenzia un grado di compromissione lieve-moderato In 25 di questi pazienti è stata, successivamente, effettuata terapia antivirale combinata con Peg-Interferone alfa 2a, alla dose di $135 \mathrm{mcg} / \mathrm{settimana}$, e Ribavirina, alla dose di $200 \mathrm{mg} / \mathrm{die}$, per 24 o 48 settimane, sulla base del 


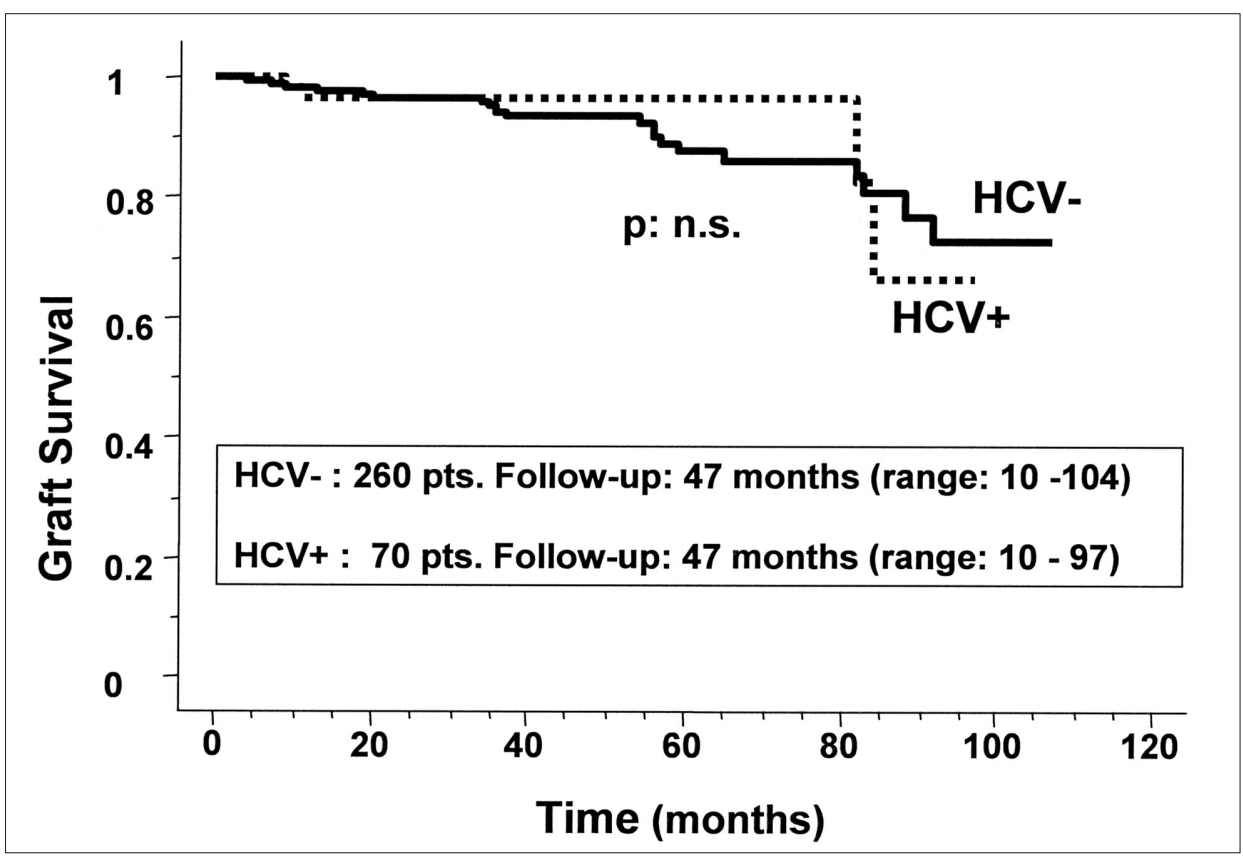

Fig. 2

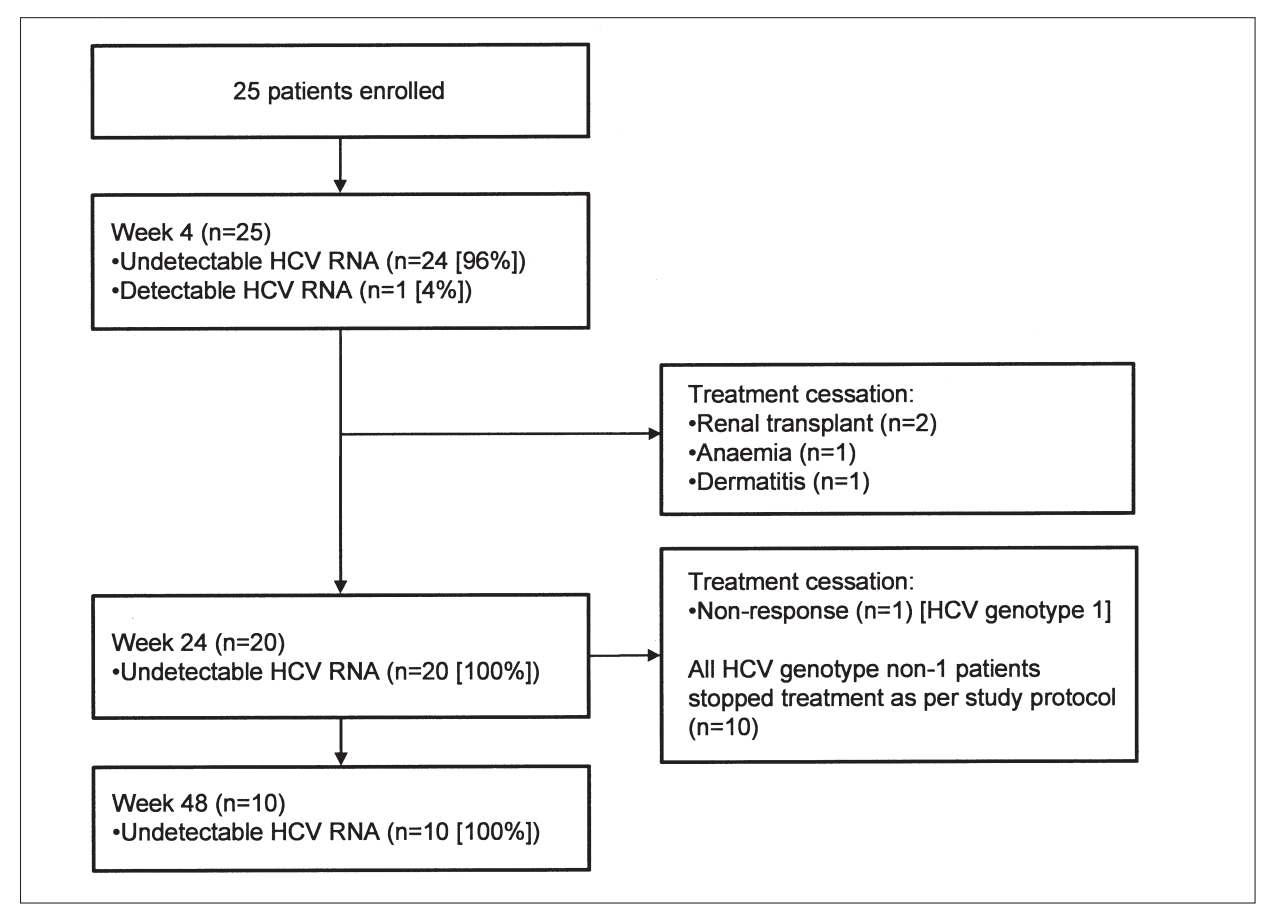

Fig. 3

genotipo coinvolto (Fig. 3). In 24 pazienti $(96 \%)$ si è ottenuta una risposta virologica sostenuta (RSV). Il tasso di drop out è risultato relativamente basso con soli 5 pazienti (14\%) che non pur avendo negativizzato la viremia, hanno ricevuto un trapianto renale prima del termine della terapia prevista. L'elevata prevalenza di potenziali riceventi $\mathrm{HCV}+(\mathrm{RHCV}+)$ in LAT ha determinato, nel tempo, la necessità di utilizzare, per questi riceventi, donatori $\mathrm{HCV}+(\mathrm{DHCV}+)$, anche allo scopo di incrementare il pool di donatori. In quest'ottica, il Centro nazionale Trapianti ha avviato in Italia un programma sperimentale di trapianto renale da $\mathrm{DHCV}+$ a RHCV+, al quale il nostro Centro ha aderito immediatamente, dato l'elevato numero di RHCV+ presenti nella nostra lista regionale. Dal novembre 2002 al dicembre 2005, abbiamo eseguito 28 trapianti di rene in RHCV+ da 14 DHCV+ (gruppo I). Accanto a questi, abbiamo effettuato, nello stesso periodo di riferimento, 32 trapianti in RHCV+ da DHCV- (gruppo II). Questi due gruppi di pazienti sono stati utilizzati per analizzare l'impatto, a breve termine, del trapianto renale $\mathrm{DHCV}+/ \mathrm{RHCV}+$ sull'outcome del graft e del paziente. La mediana di follow-up per il gruppo I era di 10 mesi. L'età media dei donatori e dei riceventi era sovrapponibile nei due gruppi, così come la media dei MM HLA e il tempo di ischemia fredda. La terapia immunosoppressiva era uguale in tutti i pazienti del gruppo I (Tacrolimus e Steroidi) mentre quella dei pazienti del gruppo II consisteva in Ciclosporina, Micofenolato mofetile e Steroidi. La funzione del graft, espressa come creatinina sierica a 1,3 e 6 mesi post-trapianto, non mostrava alcuna differenza statisticamente significativa nei due gruppi $(1.9 \pm 0.8$ vs $1.7 \pm 0.6 ; 1.7 \pm 0.7$ vs $1.6 \pm 0.6 ; 1.6 \pm 0.7$ vs $1.5 \pm 0.8 \mathrm{mg} / \mathrm{dL}$, rispettivamente). Nel periodo di osservazione una proteinuria $(>2 \mathrm{~g} / 24 \mathrm{~h})$ a insorgenza precoce (entro il $3^{\circ}$ mese post-trapianto) si riscontrava nel $13.3 \%$ e nel $9.2 \%$ dei pazienti, rispettivamente del I e del II gruppo, senza alcuna differenza statisticamente significativa nei due gruppi, al pari dell'ipertransaminasemia (ALT $\geq 2 x$ dei valori normali) rilevata, rispettivamente, nel $13.3 \%$ e nel 9.7\% dei pazienti dei due gruppi. La prevalenza di viremia (HCV RNA+) nel post-trapianto era dell' $85 \%$ nei pa- 


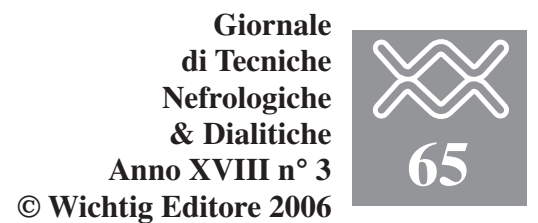

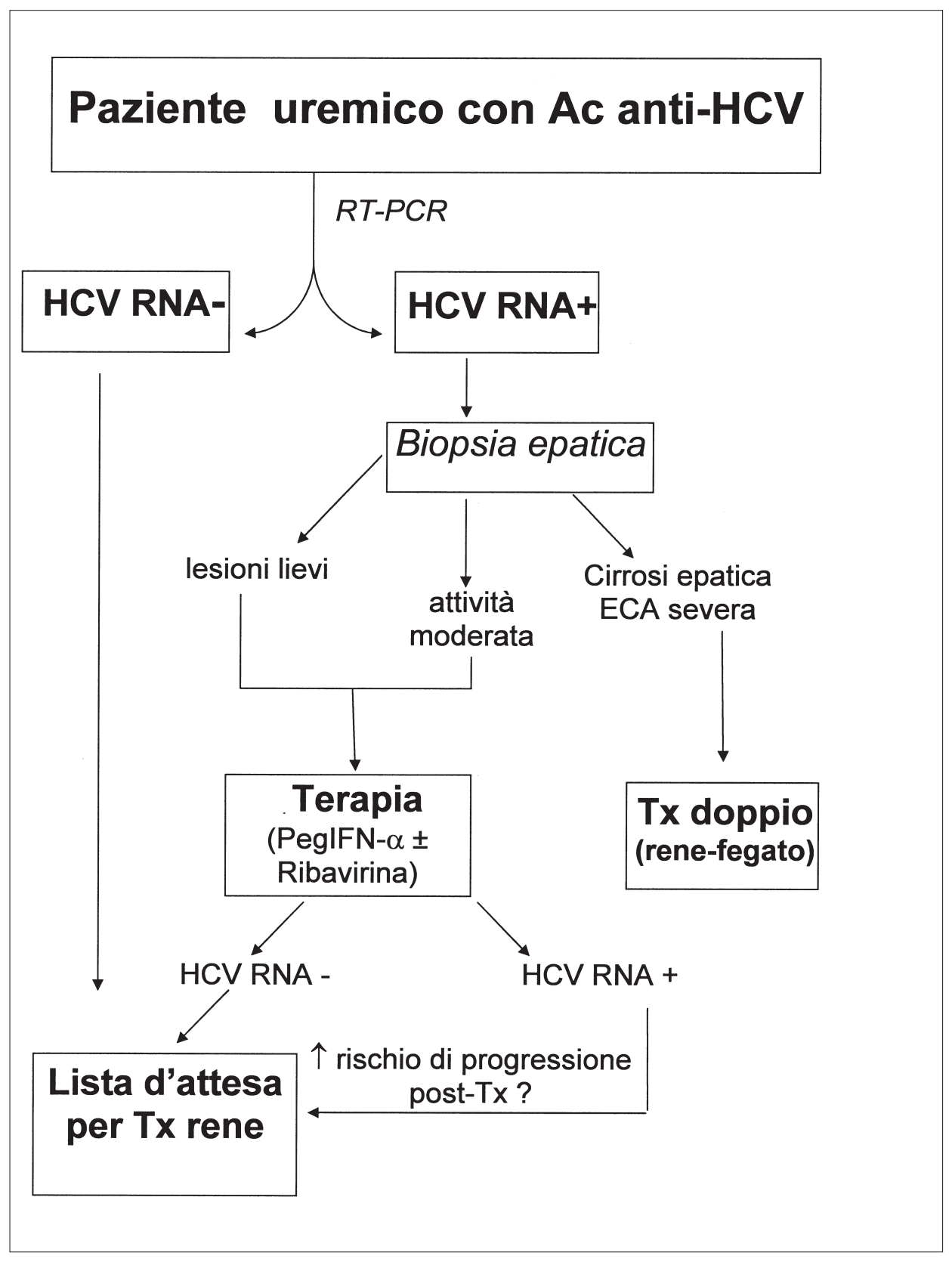

Fig. 4

zienti del gruppo I e del 59\% nei pazienti del gruppo II ( $p=n . s$.$) e in nes-$ suno dei pazienti da DHCV+ con mismatch del genotipo si osservava una superinfezione da virus del donatore, nel corso del follow-up. La sopravvivenza del graft e del paziente, a 6 mesi dal trapianto, era del 93\% nel gruppo I e del 97\% nel gruppo II senza alcuna differenza statisticamente significativa.
Per tutti i pazienti DHCV+/RHCV+, abbiamo, poi, definito un protocollo di valutazione della epatopatia $\mathrm{HCV}$ correlata. A un anno dal trapianto, 6 dei 28 pazienti $\mathrm{DHCV}+/ \mathrm{RHCV}+$ sono stati sottoposti ad agobiopsia epatica. In 5 pazienti, lo score istologico e la persistente positività ad elevato titolo dell'HCV RNA, accompagnata, in alcuni casi, da un incremento significativo delle transaminasi, hanno deter- minato la necessità di intraprendere un trattamento antivirale combinato con Peg-Interferone alfa 2a e Ribavirina, per un ciclo continuativo di 6 mesi. In tutti i soggetti, si è assistito a una rapida (entro la IV settimana di trattamento) negativizzazione dell'HCV RNA e rientro degli indici di funzione epatica, laddove si era verificato un incremento. Due pazienti non hanno completato lo schema terapeutico previsto: uno per insorgenza, a 4 settimane dall'inizio del trattamento, di rigetto acuto umorale; l'altro per grave tossicità midollare (leucopenia, piastrinopenia). Entrambi avevano, comunque, realizzato una clearance completa della viremia. Alla sospensione del trattamento, si è assistito, tuttavia, a un graduale incremento della stessa. Negli altri pazienti, il trattamento è stato ben tollerato, con realizzazione di RVS. $\mathrm{Al}$ momento attuale, pertanto, riteniamo possibile proseguire il protocollo sui pazienti RHCV+/DHCV+, con possibilità di effettuare una terapia specifica, in accordo con le indicazioni cliniche e istologiche. Una implementata esperienza e un prolungato follow-up sono, comunque, necessari per validare tale strategia quale idoneo strumento di trattamento.

A conclusione di quanto qui illustrato relativamente alla nostra esperienza, risulta evidente che, laddove possibile, il trattamento antivirale andrebbe effettuato già in fase di inserimento in lista, allorquando la tolleranza risulta migliore e il grado di risposta clinica superiore (Fig. 4). Rimane, a questo punto, ancora controversa e possibile fonte di ulteriori dibattiti in ambito trapiantologico, l'opportunità o meno che un potenziale ricevente $\mathrm{HCV}+$ che abbia realizzato una completa negativizzazione dell'HCV RNA, vada incluso nel programma di trapianto da $\mathrm{DHCV}+$, soprattutto in considerazione del fatto che, in corso di donazione, non sempre risulta possibile ottenere dei dati di viremia del potenziale donatore. 